\title{
UNIVERSAL QUANTUM COMPUTATION IN A HIDDEN BASIS
}

\author{
L. M. Ioannou ${ }^{1 \text { 田 and M. Mosca }}{ }^{1,2}$ 田 \\ ${ }^{1}$ Institute for Quantum Computing, University of Waterloo, \\ 200 University Avenue, Waterloo, Ontario, N2L 3G1, Canada \\ ${ }^{2}$ Perimeter Institute for Theoretical Physics \\ 31 Caroline Street North, Waterloo, Ontario, N2L 2Y5, Canada
}

Let $|\mathbf{0}\rangle$ and $|\mathbf{1}\rangle$ be two states that are promised to come from known subsets of orthogonal subspaces, but are otherwise unknown. Our paper probes the question of what can be achieved with respect to the basis $\{|\mathbf{0}\rangle,|\mathbf{1}\rangle\}^{\otimes n}$ of $n$ logical qubits, given only a few copies of the unknown states $|\mathbf{0}\rangle$ and $|\mathbf{1}\rangle$. A phase-invariant operator is one that is unchanged under the relative phase-shift $|\mathbf{1}\rangle \mapsto e^{i \theta}|\mathbf{1}\rangle$, for any $\theta$, of all of the $n$ qubits. We show that phase-invariant unitary operators can be implemented exactly with no copies and that phase-invariant states can be prepared exactly with at most $n$ copies each of $|\mathbf{0}\rangle$ and $|\mathbf{1}\rangle$; we give an explicit algorithm for state preparation that is efficient for some classes of states (e.g. symmetric states). We conjecture that certain non-phase-invariant operations are impossible to perform accurately without many copies. Motivated by optical implementations of quantum computers, we define "quantum computation in a hidden basis" to mean executing a quantum algorithm with respect to the phase-shifted hidden basis $\left\{|\mathbf{0}\rangle, e^{i \theta}|\mathbf{1}\rangle\right\}$, for some potentially unknown $\theta$; we give an efficient approximation algorithm for this task, for which we introduce an analogue of a coherent state of light, which serves as a bounded quantum phase reference frame encoding $\theta$. Our motivation was quantum-public-key cryptography, however the techniques are general. We apply our results to quantum-public-key authentication protocols, by showing that a natural class of digital signature schemes for classical messages is insecure. We also give a protocol for identification that uses many of the ideas discussed and whose security relates to our conjecture (but we do not know if it is secure).

\section{Introduction}

We consider a new quantum-information-theoretic problem; let us first define the problem and then summarize our results and their significance.

Suppose $\mathcal{S}$ is a $d$-dimensional complex vector space with computational basis $B=\{|i\rangle: i=$ $0,1, \ldots, d-1\}$. Assume that we have the ability to do universal quantum computation (with respect to $B)$ in $\mathcal{S}$. Let $\mathcal{S}_{0}=\operatorname{span}\left(B_{0}\right)$ and $\mathcal{S}_{1}=\operatorname{span}\left(B_{1}\right)$ be two orthogonal subspaces of $\mathcal{S}$

\footnotetext{
*lmi@iqc.ca

${ }_{\dagger}^{\dagger}$ mmosca@iqc.ca
} 
such that

$$
\mathcal{S}=\mathcal{S}_{0} \oplus \mathcal{S}_{1}
$$

and $B=B_{0} \cup B_{1}$, where the union of the orthonormal bases $B_{0}$ and $B_{1}$ is disjoint. Assume that $B_{0}$ and $B_{1}$ are known, so that we can perform universal quantum computation with respect to each of them. For all $b \in\{0,1\}$, let $A_{b}$ be a set of pure state vectors,

$$
A_{b} \subset \mathcal{S}_{b}
$$

whose classical description is known, such that no two elements in $A_{b}$ are equal up to global phase.

Definition 1 (Hidden basis). Let $|\mathbf{0}\rangle$ be a state in $A_{0}$ and let $|\mathbf{1}\rangle$ be a state in $A_{1}$, where $A_{0}$ and $A_{1}$ are defined above. These states define a hidden (computational) basis $\{|\mathbf{0}\rangle,|\mathbf{1}\rangle\}^{\otimes n}$ of $n$ logical qubits. We call this a "hidden basis" since in general the choices of $|\mathbf{0}\rangle$ and $|\mathbf{1}\rangle$ will not be known.

Remark 1 (Notation). We use boldfaced ket-labels to denote the elements of a hidden basis.

Assuming the ability to do universal quantum computation in $\mathcal{S}^{n}:=\mathcal{S}^{\otimes n}$ (with respect to the computational basis $B^{\otimes n}$ of $n d$-dimensional qudits), we investigate the number of copies of $|\mathbf{0}\rangle$ and $|\mathbf{1}\rangle$ that are required to perform unitary operations and to prepare quantum states defined with respect to the hidden basis $\{|\mathbf{0}\rangle,|\mathbf{1}\rangle\}^{\otimes n}$. Note that the question is well defined by virtue of the known classical descriptions of $A_{0}$ and $A_{1}$, which disambiguate the global phases of the states $|\mathbf{0}\rangle$ and $|\mathbf{1}\rangle$.

In Section 2, we show that any phase-invariant (see Definition 2) unitary operator on our $n$ logical qubits is exactly implementable without requiring any copies of the states $|\mathbf{0}\rangle$ and $|\mathbf{1}\rangle$. We then show (see Theorem 1) that any phase-invariant density operator on $n$ logical qubits is exactly preparable from at most $n$ copies each of $|\mathbf{0}\rangle$ and $|\mathbf{1}\rangle$. We then give an explicit, efficient algorithm for creating symmetric states, based on Ref. [1], that easily generalizes to creating any phase-invariant state. For non-phase-invariant unitary operators, such as the logical Hadamard gate, we conjecture that a large number of copies of $|\mathbf{0}\rangle$ and $|\mathbf{1}\rangle$ is needed; we give a precise conjecture, in a simplified framework, in the Appendix. Our conjecture adds to the important discussion of what can and cannot be done in quantum mechanics. Knowing the limitations of a physical (computational) theory is intrinsically interesting, but no-go theorems can also be used as building blocks for other useful results. As an example of how one might use this conjecture for a new kind of cryptographic protocol, in Section 4 we present a cryptographic protocol for identification and explain how our conjecture relates to its security. 
Note that, in practice, performing a quantum computation in the basis $\{|0\rangle,|1\rangle\}^{\otimes n}$, for the known qubit-states $|0\rangle$ and $|1\rangle$, is actually equivalent to performing a computation in the phase-shifted basis $\left\{|0\rangle, e^{i \phi}|1\rangle\right\}^{\otimes n}$, where one replaces $|1\rangle$ with $e^{i \phi}|1\rangle$ in all the operations. For example, in optical implementations, one typically assumes that a laser outputs coherent states $\sum_{w=0}^{\infty}\left(\gamma^{w} / \sqrt{w !}\right)|w\rangle$ with a random, unknown, but consistent, phase parameter $\phi=\arg (\gamma)$ (see, e.g., Ref. 20 ). When using these coherent states to drive transformations in the qubits, this is equivalent to performing the entire computation in the basis $\left\{|0\rangle, e^{i \phi}|1\rangle\right\}^{\otimes n}$. It is essential that the experimentalist maintains a consistent phase reference for the duration of the computation, but the actual value of $\phi$ is unimportant. Thus, in Section 3 , we consider the problem of quantum computing with respect to the phase-shifted hidden basis $\left\{|\mathbf{0}\rangle, e^{i \theta}|\mathbf{1}\rangle\right\}^{\otimes n}$ for potentially unknown $\theta \in[0,2 \pi)$. We show (see Theorem 2) that it is possible to approximate universal quantum computation in the phase-shifted hidden basis $\left\{|\mathbf{0}\rangle, e^{i \theta}|\mathbf{1}\rangle\right\}^{\otimes n}$, given a small phase reference state encoding $\theta$ (see Definition 4) that is analogous to a coherent light state. It follows (see Corollary 6) that, by using a small number of copies of $|\mathbf{0}\rangle$ and $|\mathbf{1}\rangle$, one can prepare such a phase reference state for unknown and uniformly random $\theta$ and thus carry out approximate universal computation in the phase-shifted hidden basis $\left\{|\mathbf{0}\rangle, e^{i \theta}|\mathbf{1}\rangle\right\}^{\otimes n}$ for unknown and uniformly random $\theta$, in analogy to the optical implementation described above.

Our motivation for considering computing in a hidden basis is rooted in quantum-public-key cryptography, a framework, introduced in Ref. [3], in which the public keys are copies of a particular quantum state encoding a classical private key 3 The goal of this type of cryptography is to achieve the best of both the quantum and classical worlds: the information-theoretic security of several known quantum cryptographic protocols (e.g. quantum key distribution [4, 5] and symmetric-key message-authentication [6]) and the advantages (over symmetric-key cryptography) of a modern public-key infrastructure (see e.g. Ref. [7] for details). Unfortunately, it has been shown in Ref. [6] that it is generally impossible to sign arbitrary quantum states, which means that such a quantum public-key infrastructure may be difficult (if not impossible) to attain. Nevertheless, it is important to determine to what extent quantum-public-key cryptography is feasible.

Our focus in this paper is on authentication schemes, where the owner, Alice, of the private key attempts to prove to another party, based on the assumption that this party has an authentic copy of Alice's public key, that it is indeed Alice who constructed a certain message (in the case of a digital signature scheme) or participated in a particular interaction (in the case of an identification scheme). The only known secure quantum-public-key signature scheme is the one-time digital signature scheme for classical messages in Ref. [3]; the scheme is one-time

\footnotetext{
${ }^{3}$ Note that the number of copies in public circulation must be limited, so that an adversary at the very least cannot take all the copies, measure them, and get a sufficiently good estimate of the private key.

${ }^{4}$ Such an interaction is assumed not to be susceptible to a man-in-the-middle attack [7].
} 
in that it can only be used to sign one message securely before the public keys need to be refreshed. A natural next step is thus to find a "reusable" quantum digital signature scheme for classical messages or prove none exists. In the context of authentication schemes, we define reusable to mean that Alice can use the same private key to sign many different messages or prove her identity many times, but a fresh copy of the public key is needed for each verification instance. In Section 4, we describe a rather natural and general cryptographic framework, based on hidden bases, that may be suitable for reusable quantum-public-key authentication schemes. We show (see Corollary 9) how our abovementioned state preparation result is a cryptanalytic tool, rendering insecure a class of quantum digital signature schemes within the framework, thus effectively extending the original no-go theorem for quantum digital signatures in Ref. [6]. Finally, in an attempt to stimulate further research in reusable quantum-public-key authentication schemes, we give a protocol for identification that uses many of the ideas discussed (but we do not know if the protocol is secure).

\section{Phase-invariant operators}

Let $\mathcal{H}$ denote the span of $\{|\mathbf{0}\rangle,|\mathbf{1}\rangle\}$; thus, $\mathcal{H}^{n}:=\mathcal{H}^{\otimes n}$ denotes the span of the hidden basis for $n$ logical qubits:

$$
\mathcal{H}^{n}=\operatorname{span}\left(\{|\mathbf{0}\rangle,|\mathbf{1}\rangle\}^{\otimes n}\right) .
$$

For any bit-string $y=y_{1} y_{2} \cdots y_{n} \in\{0,1\}^{n}$, let

$$
|\mathbf{y}\rangle:=\left|\mathbf{y}_{\mathbf{1}}\right\rangle\left|\mathbf{y}_{\mathbf{2}}\right\rangle \cdots\left|\mathbf{y}_{\mathbf{n}}\right\rangle .
$$

For any $\theta \in[0,2 \pi]$, let $U(\theta)$ be the phase-shift by $\theta$ (with respect to the basis $\{|\mathbf{y}\rangle: y \in$ $\left.\{0,1\}^{n}\right\}$ ) operator on $\mathcal{H}^{n}$ such that

$$
U(\theta):|\mathbf{y}\rangle \mapsto e^{i H(y) \theta}|\mathbf{y}\rangle,
$$

where $H(y):=\sum_{j} y_{j}$ is the Hamming weight of $y$.

Definition 2 (Phase invariant). Let $T$ be any operator on $\mathcal{H}^{n}$. Then $T$ is phase(-shift) invariant (with respect to $\left\{|\mathbf{y}\rangle: y \in\{0,1\}^{n}\right\}$ ) if and only if

$$
U(\theta) T U(\theta)^{\dagger}=T
$$

for all $\theta \in[0,2 \pi]$. 
Define the weight of $|\mathbf{y}\rangle$ to be $H(y)$, and define the weight-w subspace of $\mathcal{H}^{n}$ as

$$
\mathcal{H}_{w}^{n}:=\operatorname{span}\left(\left\{|\mathbf{x}\rangle \in \mathcal{H}^{n}: H(x)=w\right\}\right) .
$$

Fact 2. A linear operator $T$ on $\mathcal{H}^{n}$ is phase invariant if and only if it is block diagonal with respect to the decomposition $\mathcal{H}^{n}=\oplus_{w=0}^{n} \mathcal{H}_{w}^{n}$.

Proof. Writing $T=\sum_{y, z} T_{y, z}|\mathbf{y}\rangle\langle\mathbf{z}|$ and $U(\theta)=\sum_{x} e^{i H(x) \theta}|\mathbf{x}\rangle\langle\mathbf{x}|$, it is easy to show that

$$
U(\theta) T U(\theta)^{\dagger}=\sum_{y, z} e^{i \theta(H(y)-H(z))} T_{y, z}|\mathbf{y}\rangle\langle\mathbf{z}|
$$

and thus Eq. (6) may be rewritten

$$
T_{y, z}=e^{i \theta(H(y)-H(z))} T_{y, z}, \text { for all } y, z \in\{0,1\}^{n} .
$$

If $T$ is block diagonal, then the equality in Eq. (9) holds for all $\theta$ when $H(y) \neq H(z)$ because $T_{y, z}=0$; this equality always holds for all $\theta$ when $H(y)=H(z)$. To prove the other direction, note that, if $H(y) \neq H(z)$ and Eq. (9) holds for all $\theta$, then $T_{y, z}$ must be zero (otherwise one could divide both sides by $T_{y, z}$ and get a contradiction for some value of $\theta$ ).

\subsection{Exact implementation/preparation of phase-invariant unitary/density operators}

The following lemma implies that, despite our limited knowledge about $|\mathbf{0}\rangle$ and $|\mathbf{1}\rangle$, we can exactly implement any phase-invariant unitary operator $V$ on $\mathcal{H}^{n}$, given its matrix (explicitly) with respect to the hidden basis. The lemma guarantees that we can find a matrix representation of $V$ with respect to the computational basis $B^{\otimes n}$ of $\mathcal{S}^{n}$, and then use this to effect $V$ on $\mathcal{H}^{n}$; this implementation of $V$ is algorithmically exact (though, in practice, error correction would likely need to be used; we assume perfect quantum channels throughout this paper).

Lemma 3. Given the matrix representation of a phase-invariant unitary operator $V$ on $\mathcal{H}^{n}$ with respect to the hidden basis $\{|\mathbf{0}\rangle,|\mathbf{1}\rangle\}^{\otimes n}$, one can compute the matrix representation of an operator $V^{\prime}$ on $\mathcal{S}^{n}$ with respect to the computational basis $B^{\otimes n}$, such that $\left.V^{\prime}\right|_{\mathcal{H}^{n}}=V$.

Proof. Since $V$ is phase invariant, Fact 2 implies it is block diagonal with respect to $\oplus_{w=0}^{n} \mathcal{H}_{w}^{n}$, and can thus be written $V=\oplus_{w=0}^{n} V_{w}$, for $V_{w}$ unitary on $\mathcal{H}_{w}^{n}$. Let $\left\{|w, z\rangle: z=1,2, \ldots,\left(\begin{array}{c}n \\ w\end{array}\right)\right\}$ be the natural ordered basis for $\mathcal{H}_{w}^{n}$, that is, $|w, z\rangle \in\{|\mathbf{0}\rangle,|\mathbf{1}\rangle\}^{\otimes n}$ for all $(w, z)$. The operator $V_{w}$ is specified by $\left(\begin{array}{l}n \\ w\end{array}\right)$ equations of the form

$$
|w, z\rangle \mapsto \sum_{k=1}^{\left(\begin{array}{c}
n \\
w
\end{array}\right)} c_{k, z}^{w}|w, k\rangle,
$$


which we can read off the given matrix for $V$. If, for each $z \in\left\{1,2, \ldots,\left(\begin{array}{c}n \\ w\end{array}\right)\right\}$, we fix $\pi_{z}^{w}$ to be any permutation on $n$ objects 5 such that

$$
\left|\pi_{z}^{w}\left(\mathbf{0}^{n-w} \mathbf{1}^{w}\right)\right\rangle=|w, z\rangle
$$

we can rewrite Eq. (10) as

$$
\left.\left.\mid \pi_{z}^{w}\left(\mathbf{0}^{n-w} \mathbf{1}^{w}\right)\right)\right\rangle \mapsto \sum_{k=1}^{\left(\begin{array}{c}
n \\
w
\end{array}\right)} c_{k, z}^{w}\left|\pi_{k}^{w}\left(\mathbf{0}^{n-w} \mathbf{1}^{w}\right)\right\rangle
$$

Let $S_{w}^{n}$ denote the "weight- $w$ " subspace of $S^{n}$ :

$$
S_{w}^{n}:=\operatorname{span}\left\{\left|\pi_{z}^{w}(c)\right\rangle \in S^{n}: z \in\left\{1,2, \ldots,\left(\begin{array}{c}
n \\
w
\end{array}\right)\right\}, c \in\left(B_{0}\right)^{n-w} \times\left(B_{1}\right)^{w}\right\} .
$$

It suffices to show how to compute the matrix representation with respect to $B^{\otimes n}$ of a unitary operator $V_{w}^{\prime}$ on $S_{w}^{n}$ such that $\left.V_{w}^{\prime}\right|_{\mathcal{H}_{w}^{n}}=V_{w}$, for each $w$; thus, fix $w$.

Assuming $d_{0}:=\operatorname{dim} \mathcal{S}_{0}$ and $d_{1}:=\operatorname{dim} \mathcal{S}_{1}$, we can substitute into Eq. (12) the two equations

$$
|\mathbf{0}\rangle:=\sum_{i=1}^{d_{0}} \alpha_{i}\left|a_{i}\right\rangle, \quad|\mathbf{1}\rangle:=\sum_{j=1}^{d_{1}} \beta_{j}\left|b_{j}\right\rangle,
$$

where $B_{0}=\left\{\left|a_{i}\right\rangle\right\}_{i}$ and $B_{1}=\left\{\left|b_{j}\right\rangle\right\}_{j}$, and get, after changing the order of summations,

$$
\begin{gathered}
\sum_{i_{1}, \ldots, i_{n-w}} \sum_{j_{1}, \ldots, j_{w}} \alpha_{i_{1}} \cdots \alpha_{i_{n-w}} \beta_{j_{1}} \cdots \beta_{j_{w}}\left|\pi_{z}^{w}\left(a_{i_{1}}, \ldots, a_{i_{n-w}}, b_{j_{1}}, \ldots, b_{j_{w}}\right)\right\rangle \\
\mapsto \sum_{i_{1}, \ldots, i_{n-w}} \sum_{j_{1}, \ldots, j_{w}} \alpha_{i_{1}} \cdots \alpha_{i_{n-w}} \beta_{j_{1}} \cdots \beta_{j_{w}}\left(\sum_{k=1}^{w} c_{k, z}^{w}\left|\pi_{k}^{w}\left(a_{i_{1}}, \ldots, a_{i_{n-w}}, b_{j_{1}}, \ldots, b_{j_{w}}\right)\right\rangle\right) .
\end{gathered}
$$

Consider the mapping defined by the $\left(\begin{array}{c}n \\ w\end{array}\right) d_{0}^{n-w} d_{1}^{w}$ equations of the form

$$
\left|\pi_{z}^{w}\left(a_{i_{1}}, \ldots, a_{i_{n-w}}, b_{j_{1}}, \ldots, b_{j_{w}}\right)\right\rangle \mapsto \sum_{k=1}^{\left(\begin{array}{c}
n \\
w
\end{array}\right)} c_{k, z}^{w}\left|\pi_{k}^{w}\left(a_{i_{1}}, \ldots, a_{i_{n-w}}, b_{j_{1}}, \ldots, b_{j_{w}}\right)\right\rangle
$$

\footnotetext{
${ }^{5}$ Here, the $n$ objects will be the $n$ components of a vector (that functions as the label for a ket). A binary string is considered a vector of zeros and ones.
} 
for all $\left|a_{i_{l}}\right\rangle \in\left\{\left|a_{i}\right\rangle\right\}_{i}$ (for all $\left.l=1,2, \ldots, n-w\right)$, for all $\left|b_{j_{l^{\prime}}}\right\rangle \in\left\{\left|b_{j}\right\rangle\right\}_{j}$ (for all $\left.l^{\prime}=1,2, \ldots, w\right)$, and for all $z=1,2, \ldots\left(\begin{array}{c}n \\ w\end{array}\right)$. We claim that this mapping well-defines a suitable $V_{w}^{\prime}$. Indeed, it is easy to see that the $d_{0}^{n-w} d_{1}^{w}$ subspaces (indexed by $\left(i_{1}, \ldots, i_{n-w}, j_{1}, \ldots, j_{w}\right)$ )

$$
\operatorname{span}\left\{\left|\pi_{z}^{w}\left(a_{i_{1}}, \ldots, a_{i_{n-w}}, b_{j_{1}}, \ldots, b_{j_{w}}\right)\right\rangle: z \in\left\{1,2, \ldots,\left(\begin{array}{c}
n \\
w
\end{array}\right)\right\}\right\}
$$

are mutually orthogonal, and that the mapping is unitary on each of these subspaces by unitarity of $V_{w}$. Since $\operatorname{dim} S_{w}^{n}=\left(\begin{array}{c}n \\ w\end{array}\right) d_{0}^{n-w} d_{1}^{w}$, the mapping well-defines a unitary operator on $S_{w}^{n}$. The matrix entries for $V_{w}^{\prime}$ can be read off of Eqs (17).

Lemma 3 allows us to prove the following theorem, which implies that, despite our limited knowledge of $|\mathbf{0}\rangle$ and $|\mathbf{1}\rangle$, we can prepare a copy of any phase-invariant density operator on $\mathcal{H}^{n}$, given its matrix with respect to the hidden basis.

Theorem 1 (Exact preparation of phase-invariant states). Given the matrix representation of a phase-invariant density operator $\rho$ on $\mathcal{H}^{n}$ with respect to the hidden basis $\{|\mathbf{0}\rangle,|\mathbf{1}\rangle\}^{\otimes n}$, one can prepare a copy of $\rho$ using at most $n$ copies each of $|\mathbf{0}\rangle$ and $|\mathbf{1}\rangle$.

Proof. Lemma 3 implies that, in order to prepare any phase-invariant pure state $|\phi\rangle \in \mathcal{H}_{w}^{n}$, it suffices to have $(n-w)$ copies of $|\mathbf{0}\rangle$ and $w$ copies of $|\mathbf{1}\rangle$. To see this, note that there exists a unitary operator $U_{w}$ on $\mathcal{H}_{w}^{n}$ mapping

$$
|\mathbf{0}\rangle^{\otimes(n-w)}|\mathbf{1}\rangle^{\otimes w} \mapsto|\phi\rangle
$$

and that $U_{w}$ is (trivially) phase invariant. Since $\rho$ is just a probabilistic distribution of phaseinvariant pure states (because it is block diagonal), it follows that $\rho$ is preparable using at most $n$ copies each of $|\mathbf{0}\rangle$ and $|\mathbf{1}\rangle$ (assuming one can sample from this probability distribution).

\subsection{Algorithm for exact state preparation of phase-invariant states}

Theorem 1 does not address the question of efficiency. Indeed, in some cases, the required unitary operation (denoted $U_{w}$ in the proof) is efficient, as demonstrated by the following example.

Let $\left|S_{w}^{n}\right\rangle$ be the normalized symmetric sum of all $\left(\begin{array}{c}n \\ w\end{array}\right)$ states in $\{|\mathbf{0}\rangle,|\mathbf{1}\rangle\}^{\otimes n}$ that have weight $w$ :

$$
\left|S_{w}^{n}\right\rangle:=\frac{1}{\sqrt{\left(\begin{array}{c}
n \\
w
\end{array}\right)}} \sum_{x \in\{0,1\}^{n}: H(x)=w}|\mathbf{x}\rangle .
$$

As we now explain, the algorithm for state generation in Ref. [1] can be adapted to transform

$$
|\mathbf{0}\rangle^{\otimes(n-w)}|\mathbf{1}\rangle^{\otimes w} \mapsto\left|S_{w}^{n}\right\rangle .
$$


Fix $n$ and assume $0<w<n$. Hypothetically, suppose we had a copy of $\left|S_{w}^{n}\right\rangle$ and we measured the registers one-by-one from the left in the basis $\{|\mathbf{0}\rangle,|\mathbf{1}\rangle\}$. Denote the binary outcome of measuring a register by 0 (if the register was in state $|\mathbf{0}\rangle$ ) or 1 (if the register was in state $|\mathbf{1}\rangle)$. Let $X_{i}$, for $i=1,2, \ldots, n$, denote the random variable representing the outcome of measuring register $i$ (registers are enumerated from left to right). For $j=2,3, \ldots, n$ and for any $x \in\{0,1\}^{j-1}$ and $x_{j} \in\{0,1\}$, define the probabilities

$$
\begin{aligned}
p_{x} & :=P\left(X_{1} \cdots X_{j-1}=x\right) \\
p_{x_{j} \mid x} & :=P\left(X_{j}=x_{j} \mid X_{1} \cdots X_{j-1}=x\right),
\end{aligned}
$$

where adjacent bit-values denote string-concatenation and we note that the definition of $p_{x}$ holds also for $j=n+1$. Then we have $p_{1}=w / n=1-p_{0}$ and

$$
\begin{aligned}
p_{x_{j} \mid x} & =p_{x x_{j}} / p_{x} \\
p_{1 \mid x} & =(w-H(x)) /(l-j+1) \\
& =1-p_{0 \mid x} .
\end{aligned}
$$

Define the shorthand notation, for nonnegative integers $d$ and $c \geq d$,

$$
\left|d^{\{c\}}\right\rangle:=|\mathbf{0}\rangle^{\otimes(c-d)}|\mathbf{1}\rangle^{\otimes d}
$$

We can prepare $\left|S_{w}^{n}\right\rangle$ by starting with $\left|w^{\{n\}}\right\rangle$, and then applying a sequence $U_{1}, U_{2}, \ldots, U_{n}$ of phase invariant unitary operators. The first operator will be

$$
U_{1}:\left|w^{\{n\}}\right\rangle \mapsto \sqrt{p_{0}}|\mathbf{0}\rangle\left|w^{\{n-1\}}\right\rangle+\sqrt{p_{1}}|\mathbf{1}\rangle\left|(w-1)^{\{n-1\}}\right\rangle .
$$

For each $j=2,3, \ldots, n$ and for any $x=x_{1} x_{2} \cdots x_{j-1} \in\{0,1\}^{j-1}$, we define the operators, for any $d \in\{1,2, \ldots, r\}$,

$$
U_{j}:|\mathbf{x}\rangle\left|d^{\{n-(j-1)\}}\right\rangle \mapsto|\mathbf{x}\rangle\left(\sqrt{p_{0 \mid x}}|\mathbf{0}\rangle\left|d^{\{n-j\}}\right\rangle+\sqrt{p_{1 \mid x}}|\mathbf{1}\rangle\left|(d-1)^{\{n-j\}}\right\rangle\right) .
$$

Each $U_{j}$ performs an operation similar to a root-SWAP operator 6 , controlled on registers 1 through $(j-1)$, swapping register $j$ with the next closest register to the right whose state is orthogonal to the subspace containing $|\mathbf{0}\rangle$. Our $U_{j}$ also has built into it a final phase-cleanup operation, controlled on registers 1 through $j$, which removes the imaginary factor of $i$ arising from the root-SWAP operation. We can now show that $\left|S_{w}^{n}\right\rangle=U_{n} \cdots U_{2} U_{1}\left|w^{\{n\}}\right\rangle$. A

\footnotetext{
${ }^{6}$ For $0<\alpha<1$, we can define a root-SwaP-like operator, which maps $|\mathbf{0}\rangle|\mathbf{0}\rangle \mapsto|\mathbf{0}\rangle|\mathbf{0}\rangle,|\mathbf{0}\rangle|\mathbf{1}\rangle \mapsto \alpha|\mathbf{0}\rangle|\mathbf{1}\rangle+$ $i \sqrt{1-\alpha^{2}}|\mathbf{1}\rangle|\mathbf{0}\rangle,|\mathbf{1}\rangle|\mathbf{0}\rangle \mapsto i \sqrt{1-\alpha^{2}}|\mathbf{0}\rangle|\mathbf{1}\rangle+\alpha|\mathbf{1}\rangle|\mathbf{0}\rangle$, and $|\mathbf{1}\rangle|\mathbf{1}\rangle \mapsto|\mathbf{1}\rangle|\mathbf{1}\rangle$. When $\alpha=1 / \sqrt{2}$, this is the root-SWAP operator.
} 
straightforward induction (similar to that in Ref. [1]) shows that, after $U_{j}$ is applied, the state of the $n$ registers is

$$
\sum_{x \in\{0,1\}^{j}} \sqrt{p_{x}}|\mathbf{x}\rangle\left|(w-H(x))^{\{n-j\}}\right\rangle
$$

so that, after $U_{n}$ is applied, the state is $\sum_{x \in\{0,1\}^{n}} \sqrt{p_{x}}|\mathbf{x}\rangle=\left|S_{w}^{n}\right\rangle$.

The above algorithm for creating $\left|S_{w}^{n}\right\rangle$ can be generalized to create any $|\eta\rangle \in \mathcal{H}_{w}^{n}$ (and hence any phase-invariant density operator): it is clear that it can be generalized to create any state in $\mathcal{H}_{w}^{n}$ that has real coefficients; we refer to Ref. [1] for how to create the correct phases of any complex coefficients of $|\eta\rangle$ efficiently. The general algorithm is efficient as long as the conditional probabilities $p_{x_{j} \mid x}$ are efficiently computable, as in the case of the symmetric states $\left|S_{w}^{n}\right\rangle$.

Example 1. Consider the state $\rho=\int_{0}^{2 \pi}|\varphi(\theta)\rangle\langle\varphi(\theta)| d \theta$, where

$$
|\varphi(\theta)\rangle=\left(\frac{|\mathbf{0}\rangle+e^{i \theta}|\mathbf{1}\rangle}{\sqrt{2}}\right)^{\otimes n}=\frac{1}{2^{n / 2}} \sum_{w=0}^{n} \sqrt{\left(\begin{array}{l}
n \\
w
\end{array}\right)} e^{i w \theta}\left|S_{w}^{n}\right\rangle .
$$

We see that $\rho$ is phase invariant and equal to $\sum_{w=0}^{n}\left(\begin{array}{c}n \\ w\end{array}\right) 2^{-n}\left|S_{w}^{n}\right\rangle\left\langle S_{w}^{n}\right|$. Theorem 1 implies we can prepare $\rho$ using at most $n$ copies each of $|\mathbf{0}\rangle$ and $|\mathbf{1}\rangle$, and the above algorithm implies we can do so efficiently.

\subsection{Beyond phase invariance?}

One may of course ask about unitary operators and quantum states that are not phase invariant. Regarding the former, recall the finite universal set $\{H, S, T, c-Z\}$ of gates [8], where

$$
H=\frac{1}{\sqrt{2}}\left[\begin{array}{cc}
1 & 1 \\
1 & -1
\end{array}\right], S=\left[\begin{array}{ll}
1 & 0 \\
0 & i
\end{array}\right], T=\left[\begin{array}{cc}
1 & 0 \\
0 & e^{i \pi / 4}
\end{array}\right], \quad \mathrm{c}-Z=\left[\begin{array}{cccc}
1 & 0 & 0 & 0 \\
0 & 1 & 0 & 0 \\
0 & 0 & 1 & 0 \\
0 & 0 & 0 & -1
\end{array}\right]
$$

and the matrices are defined with respect to $\{|\mathbf{0}\rangle,|\mathbf{1}\rangle\}$ (in the case of the one-qubit gates) and $\{|\mathbf{0}\rangle,|\mathbf{1}\rangle\}^{\otimes 2}$ (in the case of the $c-Z$ gate). Since $S, T$, and $c-Z$ are phase invariant, we see that it is the presumed inability to implement the Hadamard gate $H$ exactly that prevents us from performing exact universal quantum computation in the hidden basis $\{|\mathbf{0}\rangle,|\mathbf{1}\rangle\}^{\otimes n}$. Indeed, we conjecture that, for worst-case $A_{0}$ and $A_{1}$ (recall their definitions in the Introduction), a large number of copies of $|\mathbf{0}\rangle \in A_{0}$ and $|\mathbf{1}\rangle \in A_{1}$ are necessary to implement one Hadamard gate or 
to prepare one copy of $(|\mathbf{0}\rangle+|\mathbf{1}\rangle) / \sqrt{2}$, if the implementation/preparation is well approximated for every $|\mathbf{0}\rangle \in A_{0}$ and every $|\mathbf{1}\rangle \in A_{1}$; see Remark 7 and the Appendix for a precise conjecture in a simplified framework.

\section{Approximate universal computation in a hidden basis}

Recall our discussion in the Introduction, where we noted that quantum computing with respect to the hidden basis $\{|\mathbf{0}\rangle,|\mathbf{1}\rangle\}^{\otimes n}$ is equivalent to computing with respect to the phase-shifted hidden basis $\left\{|\mathbf{0}\rangle, e^{i \theta}|\mathbf{1}\rangle\right\}^{\otimes n}$, as long as $\theta$ is consistent throughout the entire computation.

Definition 3 (Quantum computation in a hidden basis). Let $\rho_{0}$ be a density operator on $\mathcal{H}^{n}$ and let $W$ be a unitary operator on $\mathcal{H}^{n}$. To carry out the quantum computation (of $\left(\rho_{0}, W\right)$ ) in the (phase-shifted) hidden basis $\left\{|\mathbf{0}\rangle, e^{i \theta}|\mathbf{1}\rangle\right\}^{\otimes n}$ means to effect the operation

$$
\rho_{0} \mapsto \rho_{0}^{\prime}:=U(\theta) W U(\theta)^{\dagger} \rho_{0} U(\theta) W^{\dagger} U(\theta)^{\dagger},
$$

given a copy of $\rho_{0}$ and a classical description of $W$. We say the computation is carried out approximately, with fidelity $\sqrt{1-\epsilon^{2}}$, if we effect an operation $\rho_{0} \mapsto \rho_{0}^{\prime \prime}$ such that $\rho_{0}^{\prime \prime}$ has fidelity $\sqrt{1-\epsilon^{2}}$ with $\rho_{0}^{\prime}$.

Remark 4 (Compatibility of phase references). In Definition 3, note that $\rho_{0}^{\prime}$ will be equivalent to $W \rho_{0} W^{\dagger}$ up to conjugation by $U(\theta)$ if $\rho_{0}$ is phase invariant. More generally, if $\rho_{0}=U(\theta) \sigma_{0} U(\theta)^{\dagger}$ for some $\theta$-independent $\sigma_{0}$ on $\mathcal{H}^{n}$, then $\rho_{0}^{\prime}$ will be equivalent to $W \sigma_{0} W^{\dagger}$ up to conjugation by $U(\theta)$. The latter condition (while including all phase-invariant $\rho_{0}$, in which case $\rho_{0}=\sigma_{0}$ ) includes any non-phase-invariant $\rho_{0}$ that is (somehow) already defined with respect to the phaseangle $\theta$, e.g., $\rho_{0}=\left(\left(|\mathbf{0}\rangle+e^{i \theta}|\mathbf{1}\rangle\right)\left(\langle\mathbf{0}|+e^{-i \theta}\langle\mathbf{1}|\right) / 2\right)^{\otimes n}$ (corresponding to $\sigma_{0}=((|\mathbf{0}\rangle+|\mathbf{1}\rangle)(\langle\mathbf{0}|+$ $\left.\langle 1|) / 2)^{\otimes n}\right)$.

Note that we have not unnecessarily complicated Definition 3 by including a notion of measurement: any measurement can be expressed as an extra unitary operation (on a possibly larger space) plus a projective measurement in the hidden computational basis $\{|\mathbf{0}\rangle,|\mathbf{1}\rangle\}^{\otimes n}$; the extra unitary operation may be absorbed into $W$ and the projective measurement easily simulated by measuring with respect to the computational basis of $\mathcal{S}^{n}$.

We now show how we can achieve approximate universal quantum computation in the hidden basis $\left\{|\mathbf{0}\rangle, e^{i \theta}|\mathbf{1}\rangle\right\}^{\otimes n}$ efficiently, given a phase reference state encoding $\theta$ that is used in an analogous manner to how coherent light states are used to drive qubit transformations in an optical implementation of a quantum computer.

Recalling the discussion in Section 2.3, we note that the set $C_{\theta}:=\left\{H_{\theta}, S, T, c-Z\right\}$ is universal for quantum computation with respect to the phase-shifted hidden basis $\left\{|\mathbf{0}\rangle, e^{i \theta}|\mathbf{1}\rangle\right\}^{\otimes n}$, where 
the phase-shifted Hadamard gate is defined as

$$
H_{\theta}:=\frac{1}{\sqrt{2}}\left[\begin{array}{cc}
1 & e^{-i \theta} \\
e^{i \theta} & -1
\end{array}\right],
$$

where the matrix is with respect to the hidden basis $\{|\mathbf{0}\rangle,|\mathbf{1}\rangle\}$. Since the other gates in $C_{\theta}$ are phase invariant, it thus suffices that we show how to implement (approximately) the gate $H_{\theta}$ many times, each time on an arbitrary input, and each time with respect to the same value of unknown and uniformly random $\theta \in[0,2 \pi)$. We can actually implement, for any $\alpha \in[0,1]$, the generalized phase-shifted Hadamard gate

$$
\begin{aligned}
H_{\theta}(\alpha):|\mathbf{0}\rangle & \mapsto \alpha|\mathbf{0}\rangle+\sqrt{1-\alpha^{2}} e^{i \theta}|\mathbf{1}\rangle \\
e^{i \theta}|\mathbf{1}\rangle & \mapsto \sqrt{1-\alpha^{2}}|\mathbf{0}\rangle-\alpha e^{i \theta}|\mathbf{1}\rangle .
\end{aligned}
$$

Just as before, we will make use of phase-invariant root-SWAP-like operations, which introduce imaginary $i$ factors; thus, it will be more convenient to directly implement the gate,

$$
\begin{aligned}
G_{\theta}(\alpha):|\mathbf{0}\rangle & \mapsto \alpha|\mathbf{0}\rangle+\sqrt{1-\alpha^{2}} i e^{i \theta}|\mathbf{1}\rangle \\
|\mathbf{1}\rangle & \mapsto \sqrt{1-\alpha^{2}} i e^{-i \theta}|\mathbf{0}\rangle+\alpha|\mathbf{1}\rangle,
\end{aligned}
$$

and then we have, for example, $Z S G_{\theta}(\alpha) S Z=H_{\theta}(\alpha)$, where $Z$ is the (phase-invariant) Pauli- $Z$ gate

$$
Z=\left[\begin{array}{cc}
1 & 0 \\
0 & -1
\end{array}\right]
$$

and $S$ is defined in Eq. (32). Also, for clarity of exposition, we will assume $\alpha=1 / \sqrt{2}$ (but we will indicate in footnotes how the procedure is modified for general $\alpha$ ). Let

$$
G_{\theta}:=G_{\theta}(1 / \sqrt{2}) .
$$

Consider how one might effect the gate $G_{\theta}$, given some phase reference state that encodes $\theta$ and presumably depends on $|\mathbf{0}\rangle$ and $|\mathbf{1}\rangle$. What form could such a state take? Inspired by a coherent light state, $\sum_{w=0}^{\infty}\left(\gamma^{w} / \sqrt{w !}\right)|w\rangle$, we make the following definition.

Definition 4 (Phase reference state). For any $\theta \in[0,2 \pi)$ and positive integer $t$, a phase reference state (having size $t$ and encoding $\theta$ ) is

$$
\left|\Psi_{\theta}^{t}\right\rangle:=\sum_{w=1}^{t} e^{i w \theta}\left|w^{\{t\}}\right\rangle,
$$

where $\left|w^{\{t\}}\right\rangle$ is defined in Eq. (27). 
Each occurrence of $|\mathbf{1}\rangle$ (respectively, $|\mathbf{0}\rangle$ ) in the state $\left|w^{\{t\}}\right\rangle$ is analogous to one photon (respectively, one vacuum). Thus, it will be convenient to refer to the state $\left|w^{\{t\}}\right\rangle$ as a 1-number state, in analogy with the photon-number state $|w\rangle \cdot 7$

Remark 5 (Freedom in definition of phase reference state). Note that since we are not restricted to the standard interaction Hamiltonians present in Nature (because we are just mimicking such interactions using a universal quantum computer), our version of the coherent states, as well as how they interact with the other systems, looks slightly different; we need only mimic some of the main properties of coherent light states, for example, that the argument (phase angle) of successive coefficients scales linearly with the photon number. In general, to achieve an efficient approximation, there is some freedom in the choice of the moduli of the coefficients of our phase reference states. For clarity of presentation, we have chosen to use the simplest coefficients, with constant modulus. However, one could specify a cost function and optimize the moduli accordingly, with modest gain in the quality of the approximation 8

Define the phase-invariant controlled-root-SwAP gate $U$, which, for fixed $t$, acts on $(t+1)$ equally-sized registers, and in particular operates as follows:

$$
\begin{aligned}
U:|\mathbf{0}\rangle\left|a^{\{t\}}\right\rangle & \mapsto|\mathbf{0}\rangle\left|a^{\{t\}}\right\rangle+i|\mathbf{1}\rangle\left|(a-1)^{\{t\}}\right\rangle \\
|\mathbf{1}\rangle\left|b^{\{t\}}\right\rangle & \mapsto i|\mathbf{0}\rangle\left|(b+1)^{\{t\}}\right\rangle+|\mathbf{1}\rangle\left|b^{\{t\}}\right\rangle,
\end{aligned}
$$

for all $a=1,2, \ldots, t$ and all $b=0,1, \ldots,(t-1) \cdot 9 \quad$ Let $|\phi\rangle$ be an arbitrary one-logical-qubit pure state to which we want to apply $G_{\theta}$. Applying $U$ to $|\phi\rangle\left|\Psi_{\theta}^{t}\right\rangle$, the resulting state contains the term

$$
\sqrt{\frac{t-2}{t}}\left(G_{\theta}|\phi\rangle\right)\left(\frac{1}{\sqrt{t-2}} \sum_{w=2}^{t-1} e^{i \theta w}\left|w^{\{t\}}\right\rangle\right) .
$$

\footnotetext{
${ }^{7}$ In the right-hand side of Eq. (41), we could have had the summation start at $w=0$; we choose $w=1$ to make the analysis that follows cleaner, while not significantly affecting the quality of the approximation.

${ }^{8}$ States that are similar to our coherent-state analogues $\left|\Psi_{\theta}^{t}\right\rangle$ have independently been used in Ref. 99. In their application, the phase parameter is not an issue. Rather, they use this property of coherent states behaving more and more "classically", i.e. with less and less disturbance to the coherent state, as the size $t$ of the coherent state gets larger. In their case, a larger coherent state corresponds to a larger amount of shared entanglement, and they used this to show that more entanglement always improves the success probability of their protocol. Generally, all such states are forms of "embezzling" states [10, 11].

${ }^{9}$ Here is a complete description of $U$ : If the registers are enumerated $0,1, \ldots, t$ starting from the left, then $U$ applies the root-SWAP to registers 0 and $j$ (for $j \geq 1$ ) exactly when either registers 0 through $(j-1$ ) are in the state subspace containing $|\mathbf{0}\rangle$ and registers $j, \ldots, t$ are in the subspace containing $|\mathbf{1}\rangle$ or registers 1 through $j$ are in the subspace containing $|\mathbf{0}\rangle$ and registers 0 and $j+1, \ldots, t$ are in the subspace containing $|\mathbf{1}\rangle$ (and, otherwise, $U$ acts as the identity operator). In the case of general $\alpha$, use the root-swAP-like operator (defined in a previous footnote) instead of the root-SWAP.
} 
The other ("junk") terms are of 1-number 0, 1, and $t$. Note that terms of different 1-number are orthogonal. Recall that it suffices to compute the minimum gate fidelity over all pure inputs $|\phi\rangle$, because of the joint concavity of the fidelity [8]. Thus, the $G_{\theta}$ gate was effectively applied with minimum fidelity $\sqrt{(t-2) / t}$, and the rightmost $(t+1)$ registers have this same fidelity with the state $\left|\Psi_{\theta}^{t}(1)\right\rangle$, where we define

$$
\left|\Psi_{\theta}^{t}\left(i^{\prime}\right)\right\rangle:=\left(1 / \sqrt{t-2 i^{\prime}}\right) \sum_{w=1+i^{\prime}}^{t-i^{\prime}} e^{i \theta w}\left|w^{\{t\}}\right\rangle
$$

for $i^{\prime} \geq 0$. To implement a second $G_{\theta}$ gate, we use these same rightmost $t$ registers (whose state is close to $\left.\left|\Psi_{\theta}^{t}(1)\right\rangle\right)$. And so on. In general, we find that $U|\phi\rangle\left|\Psi_{\theta}^{t}\left(i^{\prime}\right)\right\rangle$ equals

$$
\sqrt{\frac{t-2\left(i^{\prime}+1\right)}{t-2 i^{\prime}}}\left(G_{\theta}|\phi\rangle\right)\left|\Psi_{\theta}^{t}\left(i^{\prime}+1\right)\right\rangle
$$

plus orthogonal "junk", where $|\phi\rangle$ is, again, an arbitrary pure state of a logical qubit. The gate fidelity of the $l$ th approximation of $G_{\theta}$ is thus at least $\sqrt{(t-2 l) / t}$ (for sufficiently large $t)$. Thus, we have the following theorem:

Theorem 2 (Approximate universal quantum computation in a hidden basis). Let $\rho_{0}$ be a density operator on $\mathcal{H}^{n}$ and suppose $W$ is a unitary operator on $\mathcal{H}^{n}$ that can be decomposed into phase-invariant gates and at most $l$ Hadamard gates. Given $\epsilon>0$ and a copy of the reference state $\left|\Psi_{\theta}^{t}\right\rangle$ (defined above), one can carry out the quantum computation in the hidden basis $\left\{|\mathbf{0}\rangle, e^{i \theta}|\mathbf{1}\rangle\right\}^{\otimes n}$ of $\left(\rho_{0}, W\right)$ approximately, with fidelity $\sqrt{1-\epsilon^{2}}$, if $t \geq\left\lceil 2 l / \epsilon^{2}\right\rceil$.

We say that the approximation algorithm in Theorem 2 is efficient because the size of the phase reference state need only scale linearly with the number of Hadamard gates implemented, for constant $\epsilon$. In Section 4.2.1, we show how Theorem 2 can be applied by an adversary to mount a weak attack on the cryptographic protocol we present in Section 4.2 .

We end this section with the following corollary, which summarizes how one can use copies of $|\mathbf{0}\rangle$ and $|\mathbf{1}\rangle$ to create a phase reference state $\left|\Psi_{\theta}^{t}\right\rangle$, for unknown and uniformly random $\theta$, in order to carry out approximate universal quantum computation in a hidden basis.

Corollary 6 (Computation in a hidden basis for unknown and random $\theta$ ). Let $\rho_{0}$ be a density operator on $\mathcal{H}^{n}$ and suppose $W$ is a unitary operator on $\mathcal{H}^{n}$ that can be decomposed into phaseinvariant gates and at most $l$ Hadamard gates. Given $\epsilon>0$ and $t$ copies each of $|\mathbf{0}\rangle$ and $|\mathbf{1}\rangle$, one can carry out the quantum computation in the hidden basis $\left\{|\mathbf{0}\rangle, e^{i \theta}|\mathbf{1}\rangle\right\}^{\otimes n}$ of $\left(\rho_{0}, W\right)$ for unknown and uniformly random $\theta \in[0,2 \pi)$, i.e., effect the operation

$$
\rho_{0} \mapsto \rho_{0}^{\prime}:=\frac{1}{2 \pi} \int_{\theta} U(\theta) W U(\theta)^{\dagger} \rho_{0} U(\theta) W^{\dagger} U(\theta)^{\dagger} d \theta
$$


approximately, with fidelity $\sqrt{1-\epsilon^{2}}$, if $t \geq\left\lceil 2 l / \epsilon^{2}\right\rceil$.

Proof. Noting that

$$
\frac{1}{2 \pi} \int_{0}^{2 \pi} d \theta\left|\Psi_{\theta}^{t}\right\rangle\left\langle\Psi_{\theta}^{t}\left|=\frac{1}{t} \sum_{w=1}^{t}\right| w^{\{t\}}\right\rangle\left\langle w^{\{t\}}\right|,
$$

we can thus prepare the state $\left|\Psi_{\theta}^{t}\right\rangle$ for a uniformly random value of $\theta$ by preparing a uniformly random $\mathbf{1}$-number state, which is easy to do with $t$ copies each of $|\mathbf{0}\rangle$ and $|\mathbf{1}\rangle$. The statement then follows from Theorem 2 ,

We say that the approximation algorithm in Corollary [6 is efficient because the number of copies of $|\mathbf{0}\rangle$ and $|\mathbf{1}\rangle$ need only scale linearly with the number of Hadamard gates implemented, for constant $\epsilon$. Note that if $\rho_{0}$ is phase invariant and we are interested in measuring (after executing $W$ ) a phase-invariant Hermitian observable $M$, then we can apply Corollary 6 to carry out approximate quantum computation in a hidden basis and effectively measure the observable $M$ on the state $W \rho_{0} W^{\dagger} 10$ We hope that Corollary 6 finds application (perhaps in conjunction with Theorem 2) in interactive protocols (e.g. interactive proofs), where the parties variously create/send/receive and perform quantum operations on input states $\left(\rho_{0}\right)$, output states $\left(\rho_{0}^{\prime}\right)$, and phase reference states.

\section{Applications to public-key authentication}

Our motivation is information-theoretically-secure quantum-public-key cryptography, a framework for which was first proposed by Gottesman and Chuang in Ref. [3]; we describe an example of that framework now.

Let $A_{\delta} \subset \mathbf{C}^{M}$ be a set of superpolynomially (in $\log (M)$ ) many quantum states such that, for every distinct $|\psi\rangle$ and $|\phi\rangle$ in $A_{\delta}$,

$$
|\langle\psi \mid \phi\rangle| \leq \delta
$$

for some positive constant $\delta<1$. The states in $A_{\delta}$ are sometimes called quantum fingerprints, and explicit constructions for such $A_{\delta}$, with $\left|A_{\delta}\right| \in 2^{\Omega(M)}$, are known [12]. A (succinct) classical description of $A_{\delta}$ is published.

\footnotetext{
${ }^{10}$ We have specified that the observable $M$ be phase invariant for two reasons: (1) so that the measurement statistics from measuring $M$ on $\rho_{0}^{\prime}$ are the same as if one measured $M$ on $W \rho_{0} W^{\dagger}$; and (2) so that we can implement the measurement with no approximation error, as follows. Each block $M_{w}$ of any phase-invariant observable $M=\oplus_{w=0}^{n} M_{w}$ can be diagonalized by a phase-invariant unitary $V_{w}$. Thus, we can implement $M$ by first implementing $\oplus_{w=0}^{n} V_{w}^{\dagger}$ and then measuring in the hidden computational basis $\{|\mathbf{0}\rangle,|\mathbf{1}\rangle\}^{\otimes n}$.
} 
As part of the key generation procedure, Alice randomly chooses a $|\psi\rangle$ uniformly from $A_{\delta}$ and keeps this choice secret, but she authentically distributes (e.g., by trusted courier) a limited number of copies of $|\psi\rangle$ among several members of the public (including Bob). The classical description of $|\psi\rangle$, which can be encoded by a bit-string of length $\Theta\left(\log \left(\left|A_{\delta}\right|\right)\right)$, serves as part of the private key, while several authentic copies of the state $|\psi\rangle$ serve as part of the public key. Assuming each copy of $|\psi\rangle$ is an $O(\log (M))$-qubit state, the maximum number of bits of information one can extract from $T$ copies of $|\psi\rangle$ is $O(T \log (M))$, by the Holevo bound [13, 8]. Thus, as long as $T \ll \log \left(\left|A_{\delta}\right|\right) / \log (M)$, the part of the private key corresponding to $|\psi\rangle$ is protected even if $T$ copies of $|\psi\rangle$ exist.

The full private/public key would typically consist of several independent instances of this setup, that is, Alice independently chooses several states $|\psi\rangle \in A_{\delta}$ and distributes the corresponding copies. This naturally allows for protocols that are the composition of independent instances of an atomic protocol, or kernel, that succeeds with only a certain probability. Repeating the kernel sufficiently many times, with independent $|\psi\rangle$-values each time, can amplify the success probability to an acceptable level. In the case of authentication schemes, "to succeed" means "to correctly 'accept' or 'reject' a purported message or entity".

A more general framework can be obtained by allowing the public key to consist of additional systems that may depend on the private key; we will use this more general framework in Section 4.2 .

We are primarily interested in public-key authentication protocols — either for classical messages, as in digital signature schemes, or for entities, as in identification schemes - a general approach to which is the following. Suppose that $A_{\delta}$ also satisfies the condition

$$
\langle 0 \mid \psi\rangle=0
$$

for all $|\psi\rangle \in A_{\delta}$ and some known $|0\rangle \in \mathbf{C}^{M}$. Alice can easily create states like $|0\rangle+|\psi\rangle$ (we will sometimes omit normalization factors) and, more generally, she can perform any computation in the basis $\{|0\rangle,|\psi\rangle\}^{\otimes n}$.

Remark 7 (No-Squashing Conjecture). In general, it is not known how to perform such computations efficiently, and we conjecture that it takes superpolynomially (in $\log (M)$ ) many copies of a uniformly random $|\psi\rangle \in A_{\delta}$ to prepare even one copy of $|0\rangle+|\psi\rangle$, if the procedure is to work for all $|\psi\rangle \in A_{\delta}$. We call this the No-Squashing Conjecture. We call the task of creating $|0\rangle+|\psi\rangle$ for every $|\psi\rangle \in A_{\delta}$, given copies of $|\psi\rangle$, squashing. See the Appendix for more details about squashing and the No-Squashing Conjecture.

The hope is to use this framework, for example, for Alice to convince Bob that she has prepared some state (like a signature) that no one without full knowledge of $|\psi\rangle$ could have done with only the limited number of copies of the state $|\psi\rangle$ available. Moreover, we are interested in 
reusable schemes, by which we mean that the same private key (and corresponding public keys) can be used to identify Alice many times or sign many messages from Alice (recall that the digital signature scheme in Ref. [3] is not reusable: it can only be used to sign one message). This framework may be suitable for reusable schemes, because, as suggested by our example protocol in Section 4.2, it seems to allow protocols where Alice does not divulge (to a verifier or adversary) a significant amount of extra information about the private key (beyond that which is already available from all the copies of the public key), yet retains some advantage over the adversary.

Remark 8 (Notation). In our cryptographic setting, the unknown state $|\psi\rangle$ is now playing the role of $|\mathbf{1}\rangle$. Thus, for this section of the paper, we redefine all the objects (e.g., $\left|w^{\{t\}}\right\rangle,\left|\Psi_{\theta}^{t}\right\rangle$, $\left.\left|S_{w}^{n}\right\rangle, H\right)$ that depend on $|\mathbf{0}\rangle$ and $|\mathbf{1}\rangle$, by replacing each occurrence of $|\mathbf{0}\rangle$ with $|0\rangle$ and each occurrence of $|\mathbf{1}\rangle$ with $|\psi\rangle$.

\subsection{Insecurity of a class of digital signature schemes for classical messages}

Theorem 1 can be interpreted as a restriction on any digital signature scheme for classical messages in the above framework. Before we state the result, we give a more detailed description of such a signature scheme.

Suppose that, in the key generation procedure described in the previous section, Alice chose $K$ independent values $\left|\psi_{k}\right\rangle$ from $A_{\delta}, k=1,2, \ldots, K$. Let $x$ denote the message to be signed. We assume that the full signature state for message $x$ is a $J$-fold tensor product of states

$$
\bigotimes_{j=1}^{J} \sigma_{j}\left(\left|\psi_{k(j, x)}\right\rangle\right),
$$

where $k(j, x) \in\{1,2, \ldots, K\}$ is a publicly known function depending on the particular scheme, and each $\sigma_{j}\left(\left|\psi_{k(j, x)}\right\rangle\right)$ is a density operator on $\operatorname{span}\left(\left\{|0\rangle,\left|\psi_{k(j, x)}\right\rangle\right\}^{\otimes n}\right)$ such that the coefficients of $\sigma_{j}\left(\left|\psi_{k(j, x)}\right\rangle\right)$ with respect to the basis $\left\{|0\rangle,\left|\psi_{k(j, x)}\right\rangle\right\}^{\otimes n}$ are publicly known 11 Note that, in general, $\sigma_{j}(|\psi\rangle)$ need not equal $\sigma_{j^{\prime}}(|\psi\rangle)$ when $j \neq j^{\prime}$.

We assume further that the full verification procedure breaks up into $J$ independent procedures, each denoted $P_{j}$, one for each $\sigma_{j}\left(\left|\psi_{k(j, x)}\right\rangle\right)$. Thus, if no adversary interferes, Bob would apply the procedure $P_{j}$ to $\sigma_{j}\left(\left|\psi_{k(j, x)}\right\rangle\right.$ ) (using his copy of the public key and the message) and

\footnotetext{
${ }^{11}$ These coefficients, which may also depend on the message $x$, are known in that they do not depend on the private key (classical description of $\left|\psi_{k(j, x)}\right\rangle$ ). This allows the adversary to compute the conditional probabilities required for our state preparation algorithm of Section 2.2
} 
obtain some measurement statistics. After doing this for all $j=1,2, \ldots, J$, he would process all the statistics and determine whether to "accept" or "reject" the message-signature pair.

Corollary 9 (Insecurity of a class of digital signature schemes). Suppose there is a signature scheme with signature state and verification procedure as described above. Suppose further that an adversary can obtain $n$ copies of $\left|\psi_{k(j, x)}\right\rangle$, for all $j=1,2, \ldots, J$. Then the scheme is not information-theoretically secure if, for all $j=1,2, \ldots, J$, the procedure $P_{j}$ applied to the state $\sigma_{j}\left(e^{i \theta}\left|\psi_{k(j, x)}\right\rangle\right)$ produces the same statistics as if it were applied to $\sigma_{j}\left(\left|\psi_{k(j, x)}\right\rangle\right)$, for any $\theta \in[0,2 \pi]$.

Proof. From Theorem 1, it follows that the adversary can create the uniform mixture over $\theta \in[0,2 \pi)$ of $\sigma_{j}\left(e^{i \theta}\left|\psi_{k(j, x)}\right\rangle\right)$, because this mixture is phase invariant. The procedure $P_{j}$ applied to this mixture will also produce the same statistics as if it were applied to $\sigma_{j}\left(\left|\psi_{k(j, x)}\right\rangle\right)$, thus the scheme is not information-theoretically secure.

We note that, for example, Corollary 9 applies to any scheme such that the only public key states available for use in verification of $\sigma_{j}\left(\left|\psi_{k(j, x)}\right\rangle\right)$ are copies of $\left|\psi_{k(j, x)}\right\rangle$ (and no other state dependent on the private key) and the verification procedure uses the copies of $\left|\psi_{k(j, x)}\right\rangle$ only as input to SWAP-test:12 (or similar tests for symmetry under permutations [12, 14]). Generally, the corollary implies that the verification procedure for any secure signature scheme in this framework, where the adversary can obtain sufficiently many copies of $\left|\psi_{k(j, x)}\right\rangle$, will have to exploit the global phase of the state vector $\left|\psi_{k(j, x)}\right\rangle \in A_{\delta}$ determined by its classical description.

\subsection{Example of a cryptographic protocol in this framework}

We now give an example of a cryptographic protocol that incorporates many of the ideas we have presented. The protocol is actually a translation of the honest-verifier identification scheme of Ref. [15] into our hidden basis setting. The following is an intuitive description (adapted from Section 4.7.5.1 in Goldreich's book [16]) of how a secure identification scheme works.

Suppose Alice generates a private key and authentically distributes copies of the corresponding public key to any potential users of the scheme, including Bob. If Alice wants to identify herself to Bob (i.e. prove that it is she with whom he is communicating), she invokes

\footnotetext{
${ }^{12}$ Recall that the swap-test [12, 3] of two registers (labelled 2 and 3) in the states $|\xi\rangle_{2}$ and $|\phi\rangle_{3}$ is a measurement (with respect to the computational basis $\left\{|0\rangle_{1},|1\rangle_{1}\right\}$ ) of the control register (labelled 1 ) of the state

$$
\left(H_{1} \otimes I_{2} \otimes I_{3}\right)\left(c-\operatorname{sWAP}_{2,3}\right)\left(|0\rangle_{1}+|1\rangle_{1}\right)|\xi\rangle_{2}|\phi\rangle_{3} / \sqrt{2},
$$

where $H_{1}$ is the Hadamard gate (applied to register 1 ) and $c-\mathrm{SWAP}_{2,3}$ is the controlled-SWAP gate. The probability that the state is $|0\rangle_{1}$ immediately after the measurement — which corresponds to a pass — is $\left(1+|\langle\xi \mid \phi\rangle|^{2}\right) / 2$. When the registers 2 and 3 are in the mixed states $\rho$ and $\rho^{\prime}$, this probability is $\left(1+\operatorname{tr}\left(\rho \rho^{\prime}\right)\right) / 2$.
} 
the identification protocol by first telling Bob that she is Alice, so that Bob knows he should use the public key corresponding to Alice (assuming Bob possesses public keys from many different people). The ensuing protocol, whatever it is, has the property that the prover Alice can convince the verifier Bob (except perhaps with negligible probability) that she is indeed Alice, but an adversary Eve cannot fool Bob (except with negligible probability) into thinking that she is Alice, even after having listened in on the protocol between Alice and Bob or having participated as a (devious) verifier in the protocol with Alice several times. An honest-verifier identification protocol is only intended to be secure under the extra assumption that, whenever Eve engages the prover Alice in the protocol, Eve follows the verification protocol as if she were honest. Note that no identification protocol is secure against a person-in-the-middle attack, where Eve concurrently acts as a verifier with Alice and as a prover with Bob.

As part of the key generation procedure for our protocol, we assume Alice has chosen a random $|\psi\rangle \in A_{\delta}$ and has distributed at most $r$ copies of the state

$$
|\psi\rangle(|0\rangle+|\psi\rangle)
$$

This state is the public key for one iteration of the kernel of the protocol. The parameter $r$ is the reusability parameter, dictating the maximum number of secure uses of the scheme for a particular public key.

The kernel of our interactive protocol is the following three steps, which form a typical "challenge-response" interactive proof. If the kernel is repeated $s$ times in total, then one copy of the (full) public key (of which there are still $r$ copies in total) would be $\otimes_{i=1}^{s}\left|\psi_{i}\right\rangle\left(|0\rangle+\left|\psi_{i}\right\rangle\right)$, where the $\left|\psi_{i}\right\rangle$ are each independently and uniformly randomly picked from $A_{\delta}$ by Alice. The parameter $s$ is the security parameter, which is chosen after $r$ is fixed.

1. Bob uses $|\psi\rangle$ to create the symmetric state $\left|S_{1}^{2}\right\rangle=|0\rangle|\psi\rangle+|\psi\rangle|0\rangle$ (as shown in Section 2.2), and sends the leftmost register of this state to Alice.

2. On the received register, Alice performs the logical Hadamard gate $H$ and then measures with respect to an orthogonal basis $\{|0\rangle,|\psi\rangle, \ldots\}$. If the state of the register immediately after the measurement is $|0\rangle$, then Alice sends "0" to Bob; otherwise, Alice sends "1".

3. If Bob receives " 1 ", then he applies the $Z$ gate to the register that he kept (that contained half of the symmetric state he made in Step 1). Finally, Bob SWAP-tests this register with the register containing the authentic copy of $|0\rangle+|\psi\rangle$ (the swAP-test is defined in Section 4.1).

After the kernel is repeated $s$ times, Bob "accepts" if all the swAP-tests passed; otherwise, Bob "rejects". As a final specification for the protocol, we also stipulate that Alice not engage in 
the protocol more than $r$ times (when there are $r$ copies of the public key in circulation) for a particular value of the private key.

Before discussing the potential security of this scheme, we note that the honest protocol is correct because

$$
\left|S_{1}^{2}\right\rangle=\left(H^{-1}|0\rangle\right)(|0\rangle+|\psi\rangle)-\left(H^{-1}|\psi\rangle\right) Z^{-1}(|0\rangle+|\psi\rangle)
$$

that is, Bob's SwAP-test always passes when the prover is honest (assuming perfect quantum channels).

\subsubsection{Discussion of potential security}

Within the hidden basis cryptographic framework, we refer to as black-box attacks those attacks where Eve does not use any information about the structure of $A_{\delta}$ to help her cheat. In the following discussion, we restrict our attention to the honest-verifier setting. The following definition of "security" suffices for our discussion 13

Definition 5 (Security). An honest-verifier identification protocol (for honest prover Alice and honest verifier Bob) is secure with error $\epsilon$ if the probability that Bob "accepts" when any adversary Eve participates in the protocol as a prover is less than $\epsilon$ (assuming that, whenever Eve engages Alice in the protocol, Eve follows the verification protocol honestly).

One obvious black-box attack that Eve could perform is as follows. Eve can collect $r^{\prime}:=$ $(r-1)$ copies of $|0\rangle+|\psi\rangle$, which are in the state

$$
\left(\frac{|0\rangle+|\psi\rangle}{\sqrt{2}}\right)^{\otimes r^{\prime}}=\frac{1}{\sqrt{2^{r^{\prime}}}} \sum_{w=0}^{r^{\prime}} \sqrt{\left(\begin{array}{l}
r^{\prime} \\
w
\end{array}\right)}\left|S_{w}^{r^{\prime}}\right\rangle .
$$

Now, assume Eve performs the inverse of the phase-invariant operation given in Section 2.2 , which maps $\left|S_{w}^{r^{\prime}}\right\rangle \mapsto\left|w^{\left\{r^{\prime}\right\}}\right\rangle$ with no error. The state thus becomes

$$
\frac{1}{\sqrt{2^{r^{\prime}}}} \sum_{w=0}^{r^{\prime}} \sqrt{\left(\begin{array}{l}
r^{\prime} \\
w
\end{array}\right)}\left|w^{\left\{r^{\prime}\right\}}\right\rangle .
$$

\footnotetext{
${ }^{13}$ As in Ref. [16, our definition of "security" does not include the completeness of the protocol, which stipulates that honest Bob should always accept when the prover is honest (this is easily verified for our protocol). Our definition does not take into account that there may be many different honest provers. As well, we consider neither the parallel nor serial composability of the identification protocol. See Ref. [16] for more details in the classical case.
} 
Note that this state is similar to $\left|\Psi_{0}^{r^{\prime}}\right\rangle$, but for the coefficients, which now have non-constant moduli (see Remark 5). Thus, Eve can use this state as a phase reference in order to mimic Alice's Hadamard gate.

In the black-box (honest-verifier) setting, given any $\epsilon>0$ and $r$, there indeed exists a value of $s$ (dependent on $r$ and $\epsilon$ ) such that the protocol is secure with error $\epsilon$ (assuming perfect quantum channels). For, in this case, the protocol reduces to the honest-verifier-secure protocol of Ref. [15]. The security proof follows from the work of Bartlett et al. [2] on bounded quantum reference frames and is a formalization of the following intuition. Note that Alice always causes Bob's SWAP-test to pass. However, Eve's information about the correct reference frame is limited to her $r$ samples of it (because Eve cannot extract any further information from Alice). Since only an infinite number of samples should suffice for Eve to be able to perform a measurement in the logical Hadamard basis perfectly (as Alice can), there is always some nonzero probability that Eve causes Bob's swAP-test to fail. With sufficiently large $s$, Bob will find such a failure (except with negligible probability). It turns out that $s \in \Omega(r \log (r / \epsilon))$ suffices [15].

A security proof would of course need to consider all attacks - not just black-box ones. Note that if squashing required only a small number of copies of $|\psi\rangle$ (see Remark 7), then Eve could prepare more copies of $|0\rangle+|\psi\rangle$ to use as a phase reference for her approximate implementation of $H$. This is one way that the security of our scheme depends on the assumed difficulty of squashing (e.g., the No-Squashing Conjecture); however, even if Eve could somehow transform her copies of $|\psi\rangle$ into one or more copies of $|0\rangle+|\psi\rangle$, the parameter $s$ could be modestly increased to account for Eve's extra samples of the reference frame, assuming there exists an $s$ such that the scheme is secure for $r^{\prime}$ samples of $|0\rangle+|\psi\rangle$. The security of the scheme depends more crucially on the weaker conjecture that it is impossible to perform a measurement with respect to the logical Hadamard basis $\{|0\rangle \pm|\psi\rangle\}$ (given the limited number of copies of $|\psi\rangle$ ) much more efficiently than with our black-box reference-frame approach (this conjecture is weaker because if Eve could carry out the measurement, then she could squash).

\section{Closing Remarks}

By exploiting phase invariance and mimicking properties of coherent states of light, we have shown how to perform various computational tasks, defined with respect to a hidden computational basis $\{|\mathbf{0}\rangle,|\mathbf{1}\rangle\}^{\otimes n}$, efficiently in the required number of copies of $|\mathbf{0}\rangle$ and $|\mathbf{1}\rangle$. We have shown that such tasks, which were previously not known to be possible, have cryptological application.

We have identified several open problems, including the squashing problem and the harder problem of performing measurements with respect to the hidden Hadamard basis $\{|\mathbf{0}\rangle \pm|\mathbf{1}\rangle\}$. 
Another open problem is to investigate to what extent state preparation and universal computation are possible when the assumption that $|\mathbf{0}\rangle$ and $|\mathbf{1}\rangle$ come from known orthogonal subspaces is dropped, that is, when the only promise is that $\langle\mathbf{0} \mid \mathbf{1}\rangle=0$ with $|\mathbf{0}\rangle,|\mathbf{1}\rangle \in A$, where $A$ contains no two states that are equal up to global phase.

\section{Acknowledgements}

We thank Scott Aaronson for discussions on the squashing problem and for naming it. We thank Daniel Gottesman for discussions on signature and identity schemes. We are grateful for the referees' comments on presentation, organization, and technical points. L. M. Ioannou was supported by the EPSRC and SCALA; most of this work was done while he was at the University of Cambridge. M. Mosca was supported by NSERC, QuantumWorks, MITACS, CIFAR, CRC, ORF, OCE, Government of Canada, and Ontario-MRI.

\section{References}

[1] Phillip Kaye and Michele Mosca. Quantum networks for generating arbitrary quantum states. In International Conference on Quantum Information, OSA Technical Digest Series, page PB28, Washington, D.C., June 2001. Optical Society of America.

[2] Stephen D. Bartlett, Terry Rudolph, Robert W. Spekkens, and Peter S. Turner. Degradation of a quantum reference frame. New J. Phys., 8:58, 2006.

[3] Daniel Gottesman and Isaac L. Chuang. Quantum digital signatures, 2001. arXiv:quant-ph/0105032.

[4] D. Mayers. Unconditionally secure quantum bit commitment is impossible. Phys. Rev. Lett., 78(17):3414, 1997.

[5] Peter W. Shor and John Preskill. Simple proof of security of the BB84 quantum key distribution protocol. Phys. Rev. Lett., 85:441-444, 2000.

[6] Howard Barnum, Claude Crépeau, Daniel Gottesman, Adam Smith, and Alain Tapp. Authentication of quantum messages. In IEEE Press, editor, Proc. 43rd Annual IEEE Symposium on the Foundations of Computer Science (FOCS '02), pages 449-458, 2002.

[7] A. J . Menezes, P. van Oorschot, and S. Vanstone. Handbook of Applied Cryptography. CRC Press LLC, Boca Raton, 1996. 
[8] M. Nielsen and I. Chuang. Quantum Computation and Quantum Information. Cambridge University Press, Cambridge, 2000.

[9] Debbie Leung, Ben Toner, and John Watrous. Coherent state exchange in multi-prover quantum interactive proof systems, 2008. arXiv:0804.4118.

[10] Wim van Dam and Patrick Hayden. Universal entanglement transformations without communication. Phys. Rev. A, 67(6):060302(R), 2003.

[11] Wim van Dam and Patrick Hayden. Embezzling entangled quantum states, 2002. arXiv:quant-ph/0201041.

[12] Harry Buhrman, Richard Cleve, John Watrous, and Ronald de Wolf. Quantum fingerprinting. Phys. Rev. Lett., 87:167902, 2001.

[13] A. S. Holevo. Statistical problems in quantum physics. In Gisiro Maruyama and Jurii V. Prokhorov, editors, Proceedings of the Second Japan-USSR Symposium on Probability Theory, volume 330 of Lecture Notes in Mathematics, pages 104-119, Berlin, 1973. Springer-Verlag.

[14] Georgios M. Nikolopoulos and Lawrence M. Ioannou. Deterministic quantum-public-key encryption: Forward search attack and randomization. Phys. Rev. A, to appear.

[15] Lawrence M. Ioannou and Michele Mosca. Public-key cryptography based on bounded quantum reference frames. arXiv:0903.5156.

[16] O. Goldreich. Foundations of cryptography (Volume I): Basic tools. Cambridge University Press, Cambridge, 2001.

[17] A.Y. Shiekh. The role of quantum interference in quantum computing. International Journal of Theoretical Physics, 45(9):1646-1648, 2006.

[18] V. Bužek, M. Hillery, and F. Werner. Universal-NOT gate. J. Mod. Opt., 47, 2000.

\section{Appendix: Squashing and the No-Squashing Conjecture}

The squashing problem can be meaningfully (and nontrivially) defined for a broad class of sets of states - not just sets of type $A_{\delta}$. Let $A$ be a set of pure state vectors in $\mathbf{C}^{M}$ such that no two distinct elements in $A$ are equal up to global phase. (Formally, let $\{A(M)\}_{M=1,2, \ldots}$ be a family of sets $A(M) \subset \mathbf{C}^{M}$ of complex unit vectors expressed relative to the standard 
basis $\{|0\rangle,|1\rangle,|M-1\rangle\}$. For clarity of presentation, we usually omit the "family" notation $\left.\{\cdot\}_{M=1,2, \ldots}.\right)$ Let $\epsilon>0$ be the error tolerance parameter. The pair $(A, \epsilon)$ specifies an instance of the general squashing problem, which is to compute, for every $|\psi\rangle \in A$, a state $\rho$ such that

$$
D\left(\sigma_{0, \psi}, \rho\right) \leq \epsilon
$$

given copies of $|\psi\rangle$, where $D$ is the trace distance [8],

$$
\sigma_{0, \psi}:=(|0\rangle+|\psi\rangle)(\langle 0|+\langle\psi|)
$$

and $|0\rangle$ is some known reference state (which, in general, need not be orthogonal to all $|\psi\rangle \in A$ ). Note that the classical description of $A$ also specifies a global phase for each possible state vector $|\psi\rangle$, so the ideal target state $|0\rangle+|\psi\rangle$ is well defined. The set $A$ should be nontrivial, meaning that it should contain elements that are, pairwise, not orthogonal (as would be the case when $A=A_{\delta}$ ); this is analogous to the problem of quantum cloning, where it is trivial to clone states that are promised to come from a prescribed orthogonal set.

Define $t(A, \epsilon)$ to be the smallest $t$ for which there exists a quantum operation $Q$ such that, for all $|\psi\rangle \in A$,

$$
D\left(Q\left((|\psi\rangle\langle\psi|)^{\otimes t}\right), \sigma_{0, \psi}\right) \leq \epsilon .
$$

A quantum operation $Q$ that, for some $t$, satisfies Eq. (59) for all $|\psi\rangle \in A$ is called an $(A, \epsilon)$ squasher, because it "squashes" part of the generalized Bloch sphere towards its $|0\rangle$-pole. We view the number $t(A, \epsilon)$ as measuring the complexity of the squashing problem instance $(A, \epsilon)$. We assume all $|\psi\rangle$ in $A$ are reasonably encoded into qubits and thus take the input size of the problem to be $\log (M)$.

Our cryptographic motivation leads us to look for sets $A$ such that $t(A, \epsilon)$ is necessarily large (for sufficiently small $\epsilon$ ). For some instances of the general squashing problem, exponential lower bounds on $t(A, \epsilon)$ might be relatively easily obtainable, because it might be the case that, for subexponential values of $t$, the states $\sigma_{0, \psi}$ and $\sigma_{0, \phi}$ are further in trace distance (or, equivalently, have lower fidelity) than $|\psi\rangle^{\otimes t}$ and $|\phi\rangle^{\otimes t}$. The following example of this shows that the general squashing problem subsumes the black-box search problem (this fact has already been pointed out in Ref. [17], though the author of that paper assumes that squashing is an easy task).

Example 2. Denote by $F$ the quantum Fourier transform on $\mathbf{C}^{M}$, so that

$$
F|0\rangle=(1 / \sqrt{M}) \sum_{i=0}^{M-1}|i\rangle .
$$


For every $j=0,1, \ldots, M-1$, define the state $\left|j^{*}\right\rangle$ via

$$
\left\langle i \mid j^{*}\right\rangle= \begin{cases}+1 / \sqrt{M} & \text { if } i=j, \\ -1 / \sqrt{M} & \text { if } i \neq j\end{cases}
$$

Let $A_{2}:=\left\{F^{\dagger}\left|j^{*}\right\rangle: j=0,1, \ldots, M-1\right\}$ (note that $A_{2}$ does not have the property that $\langle 0 \mid \psi\rangle=0$ for all $|\psi\rangle \in A_{2}$ ). Noting that $\left|j^{*}\right\rangle+F|0\rangle \propto|j\rangle$, we can solve the black-box search problem with a good $\left(A_{2}, \epsilon\right)$-squasher, where $\epsilon$ is a constant (i.e., if the solution to the search problem is $j$, then the black box can be used $t$ times to make $t$ copies of $\left|j^{*}\right\rangle$ ). Thus, the well-known $\sqrt{M}$ search lower bound applies to $t\left(A_{2}, \epsilon\right)$. But this is overkill: the lower bound we get from the fact that quantum operations cannot increase trace distance is larger when $\epsilon<1 / 2$, as we now show. If $Q$ is an $\left(A_{2}, \epsilon\right)$-squasher, then we have (for $i \neq j$ )

$$
\begin{aligned}
1-2 \epsilon & \leq D\left(Q\left(\left(F^{\dagger}\left|i^{*}\right\rangle\left\langle i^{*}\right| F\right)^{\otimes t}\right), Q\left(\left(F^{\dagger}\left|j^{*}\right\rangle\left\langle j^{*}\right| F\right)^{\otimes t}\right)\right) \\
& \leq D\left(\left(\left|i^{*}\right\rangle\left\langle i^{*}\right|\right)^{\otimes t},\left(\left|j^{*}\right\rangle\left\langle j^{*}\right|\right)^{\otimes t}\right) \\
& \leq \sqrt{1-\left|\left\langle i^{*} \mid j^{*}\right\rangle\right|^{2 t}} \\
& =\sqrt{1-(1-4 / M)^{2 t}}
\end{aligned}
$$

so that $t \geq \log (4 \epsilon(1-\epsilon)) / 2 \log (1-4 / M)$ and thus, for example, $t\left(A_{2}, \frac{1}{3}, 1\right) \in \Omega(M)$. The first line follows from several applications of the triangle inequality and the fact that the trace distance between the squasher's ideal outputs is 1. In subsequent lines, we have used the well-known relationship between trace distance and fidelity (see Ref. [8]) and the power series expansion $\log (1-x)=-x-x^{2} / 2-x^{3} / 3-\cdots$ for $x \in[-1,1]$ and the fact that, for $x \leq 1 / 2$,

$$
x+x^{2} / 2+x^{3} / 3+\cdots \leq x\left(1+x+x^{2}+\cdots\right)=x(1-x)^{-1} \leq 2 x .
$$

The cryptographic framework uses sets of type $A_{\delta}$, that have, among others, the two properties defined in Eq. (49) and Eq. (50). The latter property, that $\langle 0 \mid \psi\rangle=0$ for all $|\psi\rangle$ in $A_{\delta}$, is particular to our version of the framework. The former property of pairwise $\delta$-almostorthogonality is a reasonable condition to impose on quantum-public-key cryptography in general, in order to avoid a situation where two different private keys correspond to practically indistinguishable physical scenarios. For example, suppose Alice- $x$ has private key $x$ and Alice- $y$ has private key $y$, and $x \neq y$; we would not want Alice- $y$ to be able to convince Bob that she is Alice- $x$ with significant probability. This property also rules out trivial lower-bound arguments based on distinguishability, like in Example 2, because an $\left(A_{\delta}, 0\right)$-squasher does not increase pairwise distinguishability, by which we mean that, for all distinct $|\psi\rangle$ and $|\phi\rangle$ in $A_{\delta}$,

$$
D\left(\sigma_{0, \psi}, \sigma_{0, \phi}\right) \leq D\left((|\psi\rangle\langle\psi|)^{\otimes t},(|\phi\rangle\langle\phi|)^{\otimes t}\right)
$$


for some $t \in O(\operatorname{polylog}(\mathrm{M}))$; in the particular case of $A_{\delta}$, the inequality (67) actually holds for a constant $t$ (dependent on $\delta$ ). The set $A_{2}$ in Example2 has neither of the two properties discussed in this paragraph, and we cannot see how to harness the provable difficulty of squashing $A_{2}$ for potential cryptographic application.

We now state the No-Squashing Conjecture more precisely. Call $A_{\delta}$ efficient if and only if there is a succinct description of $A_{\delta}$ (so that its classical description may be easily published) and each state in $A_{\delta}$ is efficiently computable given its classical description (i.e., although we do not bound Eve's computational power, we prefer that Alice and Bob are bounded by polynomial time). Since the trace distance between $\sigma_{0, \psi}$ and the trivially-preparable mixture $|0\rangle\langle 0|+| \psi\rangle\langle\psi|$ is $1 / 2$, we take $\epsilon<1 / 2$ in the conjecture.

Conjecture 10 (No-Squashing). There exists an efficient $A_{\delta}$ such that $t\left(A_{\delta}, \epsilon\right) \in \omega\left((\log (M))^{k}\right)$ for all constants $k>0$ and any nonnegative constant $\epsilon<1 / 2$.

Our choice of the superpolynomial bound in the conjecture corresponds to the usual meaning of "large" or "inefficient" in complexity theory. We do not claim that proving such a bound has immediate cryptographic application: recall that no result about the difficulty of squashing is sufficient for establishing the honest-verifier security of the protocol of Section 4.2.

We also leave as an open problem to find any set $A$ such that the $(A, 0)$-squasher does not increase pairwise distinguishability and is not completely positive (or prove no such $A$ exists). Note that the one-qubit universal-NOT gate [18, which maps

$$
\alpha|0\rangle+\beta|1\rangle \mapsto \beta^{*}|0\rangle-\alpha^{*}|1\rangle
$$

for any qubit state $\alpha|0\rangle+\beta|1\rangle$, does not increase pairwise distinguishability and is known to be not completely positive. 